\title{
2 Die verschiedenen Dimensionen von Bewusstsein - was bedeuten sie für uns?
}

\author{
Hinderk M. Emrich
}

\section{Einleitung}

Der Sprach- und Bewusstseinsphilosoph Ludwig Wittgenstein schreibt an einer Stelle in seinem „Blauen Buch“ (Wittgenstein 1984):

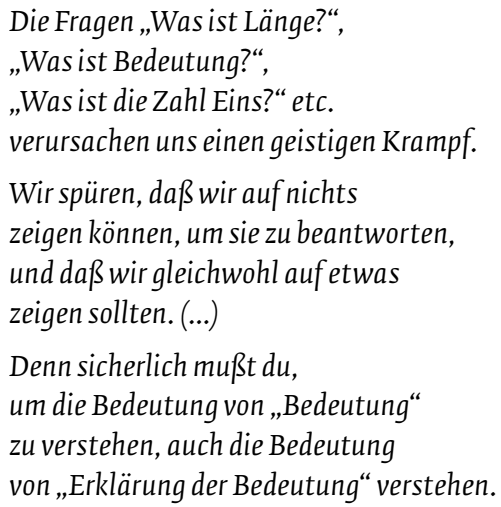

Wittgenstein fragt hier nach der „Erklärung von Bedeutung“ und analog könnte man sagen, auch die Frage nach der Erklärung von Bewusstsein ist in ähnlicher Weise mit einer Aporie, einem ,geistigen Krampf“"verbunden. Auch hier kann gefragt werden: Geht es nicht nur um das Erkennen der Bedeutung von Bewusstsein, sondern auch um die Bedeutung des „Erklärens von Bewusstsein“? 
Denn Bewusstsein hat immer mit der Frage zu tun:

Was bedeutet das, was mir bewusst wird, für mich?

In diesem Sinne lässt sich der sehr überzeugende Gedanke von Franz von Brentano und von Edmund Husserl interpretieren, dass Bewusstsein immer mit „Intentionalität“"zu tun hat, immer zu tun hat mit der Frage nach dem „etwas Meinen“, sich auf etwas beziehen, einen intentionalen Gegenstand in sich selbst und außerhalb des Subjekts quasi „aufzurufen“.

Und noch etwas möchte ich aus Ludwig Wittgensteins Philosophie hier anmerken: Er formulierte einmal: Schmerzen gibt es, sofern einer sie hat. So kann man analog formulieren:

„Bewusstsein gibt es, sofern jemand es hat.“

\section{Was ist Bewusstsein?}

Über Bewusstsein zu forschen und darüber zu diskutieren, ist eine „faszinierende Angelegenheit“, denn das Bewusstseinsphänomen enthält eine eigentümliche Besonderheit: Wir vollziehen in der Bewusstseinsforschung eine Reflexionsleistung über ein Phänomen, das die Voraussetzung darstellt für eben diese Reflexionsleistung.

Über Bewusstsein zu reden ist nur möglich unter der Voraussetzung, dass es in uns Bewusstsein gibt.

Dieses Phänomen gehört in den erkenntnistheoretischen Bereich des „anthropischen Prinzips“: Der Mensch kann sich über seine eigenen („anthropischen“) Eigenschaften nur klar werden durch die Funktionsweisen eben gerade dieser Eigenschaften. Diese wenn man so will Zirkularität des Forschungsansatzes der Bewusstseinsforschung ist auch der Ausgangspunkt der Transzendentalphilosophie Immanuel Kants, die nach den apriorischen Bedingungen der Möglichkeit von Erkenntnis fragt. Und sie ist somit die denkerische Klippe, die im Beginn jeder Bewusstseinsforschung steht.

In diesem Sinne lässt sich feststellen: Die Frage nach der Natur des Bewusstseins ist deshalb so schwer zu beantworten, weil wir selbst im Bewusstsein vorkommen. Alles, was über uns selbst, über unser Leben, über uns Menschen, über „Welt“ ausgesagt werden kann, kann nur ausgesagt werden, von unserem „Im-Bewusstsein-Stehen" aus. Bewusstsein ist also das Medium, das Elixier unseres Selbstseins, ja in diesem Sinne des Seins überhaupt. Was also kann darüber noch mehr gesagt werden als eben diese Darstellung der Qualifikation von Bewusstsein als Sein?

Die Bewusstseinstheorie, die philosophische Konzeption, die der hier dargestellten basalen kognitiven Eigenschaften von Bewusstseinsgegebenheiten am deutlichsten Rechnung trägt, ist die Transzendentalphilosophie von Immanuel Kant, insbesondere in seinem Werk „Kritik der reinen Vernunft“ (Kant 1974). Kant findet in diesem Werk einen Weg der „Ableitung“ von kognitiven Leistungen, die wir an uns selbst kennen und wahrnehmen, von bewusstseinsvorgängigen (,apriorischen“) Voraussetzungen der Bewusstseinsphänomene, nämlich die sog. „transzendentale Deduktion der reinen Verstandesbegriffe“.

Die Reflexion über die empirischen Aspekte der Bewusstseinsphänomene möchte ich einleiten mit einem Zitat des Transzendentalphilosophen Johann Gottlieb Fichte, der sich am stärksten auf Kant bezogen hat, mit Fichtes Worten:

Was war ich, ehe ich zum Bewusstsein kam? (Fichte 1979) 
Hier ist nicht nur von Bewusstsein sondern von Selbstbewusstsein die Rede; und die Frage Fichtes lautet: Bin ich ohne ein Bewusstsein meiner selbst ein „Es“, ein „Was“? Jedenfalls offenbar kein „Ich“. Und ich möchte hier ergänzend bemerken: Der Fichtesche Satz erinnert an das Wort von Sigmund Freud: Wo „Es“ war soll „Ich“ werden (Freud 1923).

Die geistig-seelische Arbeit auch in der Psychotherapie verschiebt die Grenzen zwischen dem Wach-Bewusstsein und dem Unbewussten und führt damit Klärungsprozesse, in diesem Sinne ein tieferes „Selbstbewusstsein“, herbei. Selbstbewusstsein ist hiermit also gewissermaßen die Eintrittspforte in das Reich der Personalität, der Subjektivität, des reflektierten Selbst-Seins.

\section{Bewusstsein als Rahmungs-Prozess}

Um den Bewusstseinsbegriff von seinen idealistischen Anteilen der Selbstreflexion etwas zu entlasten, wird heute vorgeschlagen, von „conscious awareness“ $z$ u reden, d.h. von aufmerksamer Bewusstheit, die das Selbstverhältnis nicht wesentlich im Blick hat. Bewusstheit erscheint damit als diffuser, globaler Sammelbegriff für die mentale Welt, in der „wir“ uns vorfinden. Und für Forschungszwecke wird dieser unterteilt in verschiedene Aspekte der phänomenalen Bewusstheit, wobei diese potenziell oder auch manifest sein kann, je nach dem Rahmen der jeweiligen Aufmerksamkeit.

Mein Konzept von Bewusstheit bezieht sich dabei auf die Verwendung konstruktivistischen Gedankengutes in der Neurobiologie. Dies in Verbindung mit einem Vorschlag, den Ervin Goffman in seinem Werk „Frame Analysis“, „Rahmenanalyse“ (Goffman 1977), gemacht hat; hier heißt es in der Einleitung:

Ich gehe davon aus, dass Menschen, die sich gerade in einer Situation befinden, vor der Frage stehen:Wasgeht hier eigentlich vor? Ob sie nun ausdrücklich gestellt wird ... oder stillschweigend... - die Frage wird gestellt und die Antwort ergibt sich daraus, wie die Menschen weiter in der Sache vorgehen.

Es geht dabei um Kontextanalyse; und in diesem Sinne könnte man sagen: Gehirne sind Bewusstheit-generierende „Kontext-Systeme“.

Goffman bezieht sich auf John Austin, der im Anschluss an Wittgenstein darauf hinweist, dass es eine schwierige Frage ist, was man mit „es geschieht wirklich“ meint:

„Ein Mensch könne zwar Unwirkliches träumen, doch dabei sei es immer noch angebracht zu sagen, er träume wirklich."

In diesem Sinne ist die von Goffman entwickelte „Rahmenanalyse“ geeignet, die durch Bewusstseinszustände gebildeten „Situationen“ adäquat zu beschreiben, wie beispielsweise diejenige eines Schachspiels:

Ein Spiel wie das Schach schafft für den, der ihm folgen kann, eine bewohnbare Welt, eine Ebene des Seins, Bewusstheit als Sein, ein Ensemble von Gestalten mit anscheinend unbegrenzt vielen verschiedenen Situationen und Handlungen, in denen sich ihre Eigenart und ihr Schicksal verwirklicht.

In diesem Sinne verwendet auch der Psychiater und Anthropologe Gregory Bateson den Begriff des „Rahmens“ zur Beschreibung von verschiedenen Bewusstseinsebenen wie denjenigen von Phantasie, Spiel und Alltagsrealität. Es ergibt sich die These, 
dass das Bewusstseinsphänomen als solches in der Sprache von „Frames“, von Rahmungen, besonders gut beschrieben werden kann, weil diese Sprache es ermöglicht, ein Bewusstseinsfeld in seiner Kontextualität zu erfassen und zu beschreiben. Es wird sich aber später zeigen, dass es möglich ist, Bewusstseinszustände auch jenseits dieser Rahmungen zu definieren, d.h. jenseits der Begrenzungen, in die wir üblicherweise die Bewusstseinsphänomene „einklammern“.

\section{Frames: Bewusstheits-Rahmungen als Horizonte}

Man könnte sagen: Die Bewusstheit zeichnet sich dadurch aus, dass es für die Inhalte seiner selbst einen Rahmen schafft, dessen Begrenzung Horizontcharakter aufweist. Die Prozesse dieses Rahmen-Schaffens, neurobiologische Prozesse, sind aber für das Bewusstsein hermetisch, verschlossen, sind bewusstseins-transzendent, worauf bereits L. Wittgenstein hinwies. „Wir“ können nur das „wie“ dieses Rahmenschaffens, dieses „Framings“ beobachten, können aber nicht zugleich diese Vorgänge, als Prozesse, als ihr „was“ verfolgen, wodurch letztlich die Leib-SeeleSpaltung als subjektives Phänomen zustande kommt. In diesem Sinne sind wir uns selbst in einem elementaren Sinne verborgen. Was am Rand dieses kognitiven Rahmens geschieht, ist ein „Hervorholen“, ein Hereinholen aus dem Bereich des Unbekannten, Unerschlossenen in den Rahmen des kognitiv Zugänglichen. Wir können uns bei dem Wie dieses Hervorholens zusehen, aber nicht das Objektive dieses Hervorholens verstehen und beschreiben. Das ist es, was der Philosoph Wolfgang Cramer meint, wenn er sagt: Bewusstsein zieht Welt aus sich heraus. Was außerhalb des Rahmens, was jenseits des Horizontes des aktualen Bewusstseins steht, kann prinzipiell als potenzielle Bewusstheit erfahrbar sein, wie durch ein Schiff, das über eine Horizontlinie fährt, aber diese Erreichbarkeit bleibt oft virtuell. Die hier (am Rahmen) vorliegenden Hemm-Prozesse beruhen auf Zensur-Mechanismen, die die Erreichbarkeit latenter Informationen, latenten Wissens in uns einschränken. Von dem Physiker Prof. Urban (Urban 1998) stammt der Satz: Information ist der Vergleich mit Vorhandenem. In diesem Sinne ist „Zensur“ dann die Limitation, die Verweigerung relevanter neuer Information, neuer derartiger Vergleiche mit derzeit Vorhandenem. Wie kommt es zu diesen Limitationen, Einschränkungen, Hemmnissen? Wie kommt es überhaupt zum „Rahmen“? In der Zensur liegt in der Regel ein Schutz des Systems vor Überlastung, vor „overload“, vor Störungen des Systems durch Informationsüberflutung, ein Schutz vor Psychose. Wo zu viele Vergleiche zugleich erfolgen sollen, entsteht Chaos. Das System wird handlungsunfähig. In psychotischen Dekompensations-Zuständen, psychotischen „Nervenzusammenbrüchen“ werden kognitive Systeme durch Mangel an Selbstlimitation quasi reizüberflutet. Es geht hier also um die Frage nach der Regulation der Weite des Rahmens, des „Frames“: Wie wird der Horizont des Bewusstseinsrahmens reguliert (s. Abb. 3)?

Ist er zu weit, so droht psychotische Dekompensation, ist er zu eng, bleibt das System unterinformiert, ist durch Informationsmangel bewusstseinsgestört. Was nun zu zeigen versucht werden soll, ist, dass Bewusstseinszustände vom bewusstseinsgenerierenden Apparat selbst intern interaktiv reguliert werden durch selbstzensurierende Mechanismen im Gehirn.

Bewusstsein als Rahmen bedeutet den Gedanken, dass es eine Art „semantische Topologie“ der Vereinbarung im Bewusstsein gibt. Was ist damit gemeint? 


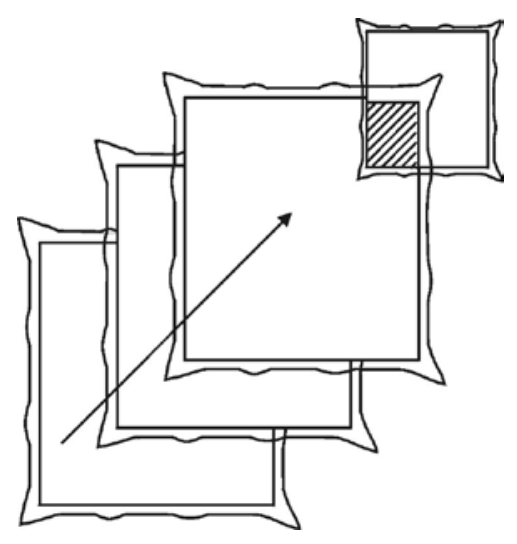

Abb. 3 Rahmenparadigma als Metapher für verschiedene Traumzustände des Bewusstseins und deren Überlagerung sowie die Gemeinsamkeit von kognitiven Gehalten im Wachbewusstsein

Nach Sigmund Freud (Freud 1914) gibt es im Bewusstseins-Apparat sehr schwergewichtige Konventionen; dies im Sinne gesellschaftlicher Normen:

- Wann und wie und warum ist etwas „Pein-lich“?

- Wieso ist etwas Pein-erzeugend und gesellschaftlich geächtet?

- „Kränkend“ und damit krank-machend?

Es wird dies Tabuisierte des Lebens ins Unbewusste verdrängt, weil es abgewehrt werden muss, weil es nicht zu meinen subjektiv erlebten und dann verdrängten Eigenschaften gehören darf, nicht so sein darf. Insofern ist das Bewusstsein nicht nur fließend, wie bei William James, als ein „stream of consciousness“(James 189o), sondern topologisch eingeengt auf bestimmte konventionell legitimierte semantische Bereiche, wie sie bei Goffman als „frames“ beschrieben worden sind, Bereiche, innerhalb derer sich die sozialen Geschehnisse abspielen dürfen. Übrigens hat die große philosophische Dichterin Virginia Woolf in ihrem faszinierenden Romanwerk „Wellen“ den „stream of consciousness“ von James eindrucksvoll beschrieben mit folgenden Worten (Woolf 1994):

„Und die Zeit“, sagte Bernard, , lässt ihren Tropfen fallen. Der Tropfen, der sich am Dach der Seele gebildet hat, fällt. Vom Dach meines Geistes läßt die Zeit, die sich formt, ihren Tropfen fallen. Letzte Woche, als ich mich rasierte, fiel der Tropfen. Ich stand mit dem Rasiermesser in der Hand da, und plötzlich wurde mir das rein Gewohnheitsmäßige meiner Handlung (das ist der Tropfen, der sich formt) bewußt, und ich beglückwünschte voll Ironie meine Hände, weil sie nicht davon abließen. Rasiert nur, rasiert, rasiert, sagte ich. Rasiert nur weiter. Der Tropfen fiel. Den ganzen Tag über begab sich, während ich arbeitete, mein Geist von Zeit zu Zeit an einen leeren Ort und sagte, ,Was ist dahin? Was ist vorbei?' Und ,Aus und vorbei', murmelte ich, aus und vorbei', mich mit Worten tröstend. Den Leuten fiel auf, wie leer mein Gesichtsausdruck war und wie ziellos mein Reden. Dieletzten Wörter meines Satzes verloren sich. Und alsich mir den Mantel zuknöpfte, um nach Hause zu gehen, sagte ich ziemlich dramatisch, , Meine Jugend ist dahin'“”

Das Phänomen der „Frames“ als einer „Rahmung von Bewusstsein“ - im Gegensatz hierzu - stellt sich als eine sehr geeignete Metapher für das bewusste „Bewusstseinsfeld“ dar. 


\section{Unsagbarkeit und Bewusstsein}

Nun gibt es eine Verstehensperspektive, von der aus das Wort „Bewusstsein“ problematisch ist. Dies aus folgendem Grunde: Das Wort Bewusstsein ist ein Substantiv, kein Adjektiv, kein Verb. (Man kann auch sagen: ein substantivistischer Begriff, aber eben nicht eine Beschreibung des Phänomens selbst, eine Art „Platzhalter“). Es wird sprachlich behandelt wie ein Gegenstand.

Nun ist aber der philosophische Einwand zu bedenken, dass Substantive Konstrukte unseres Geistes sind, die uns die sprachlichen Wirklichkeits-Verweise erleichtern, andererseits uns aber auf ein falsches Gleis führen. Einen Beitrag hierzu leistet der amerikanischen Philosoph Alan North Whitehead - er ist zugleich theoretischer Physiker gewesen. Er sagt in seinem Buch „Process and Reality“ (Whitehead 1979), dass es

- keine Naturgesetze gibt

- keine Dinge gibt.

Warum dies?

Naturgesetze sind etwas Abstraktes. Sie sind Vorschriften, eine Ordnung, die man den Dingen aufgezwungen hat, sich immer genau so und so zu verhalten. Aber ein solches Aufzwingen gibt es nicht in der Natur, sondern es gibt nur Prozesse, die Singularitäten aufweisen; unter den und den Bedingungen machen beispielsweise Protonen, Positronen, Wasserstoffmolekülionen dies und jenes.

Und mit den Dingen ist es auch nicht weit her: Hans-Peter Dürr führt immer wieder zu Recht die „Immaterialität der Materie“ aus. Er sagt: Es gibt nur „Verbundenheiten“. In diesen ereignet sich immer neues Procedieren, quasi als „Verben“.

Der Psychoanalytiker Roy Schafer sagte in seinem Buch „Eine neue Sprache für die Psychoanalyse“ (Schafer 1982): Es gibt keine „Instanzen“ in uns; es gibt nur Prozesse in uns. Diese müssen sprachlich als Verben auftreten, ausgedrückt werden.

Wenn das stimmt, gibt es kein „Bewusstsein“, es gibt nur

ein geistiges Erleben, ein Fühlen, ein Wahrnehmen, einen Meinen, ein Wollen, ein Fürchten, ein sich Ängstigen;

und dies kann latent oder manifest in uns sein. Bewusstes Bewusstsein wäre dann ein aktuales Meinen, Wollen, Fürchten; und unbewusstes Bewusstsein wäre dann nicht aktualisiertes aber latent vorhandenes Meinen, Wollen, Fürchten etc.

Aber Bewusstsein und Bewusstheit als „Instanz“ gibt es nicht, sondern nur die Lebendigkeitsvorgänge des sich auf etwas Beziehens, des etwas Meinens, des etwas Wollens etc.

Im Bewusstsein sein ist also kein „Sein“ im substantivischen Sinne (ontologisch), sondern ein Vollziehen von Erleben, als Verb, als Vorgang, als ein inneres und äußeres Vollziehen. Man kann nun allerdings die Frage nach Bewusstsein als „Sein“ und andererseits als „Prozess“ drehen und wenden wie man will: Man kommt nicht daran vorbei, dass das „im Bewusstsein Sein“ immer etwas „meint“. Auch Sprache meint etwas: So sind Sprache und Bewusstsein sehr eng verwandt. Sprache als Paralleluniversum zu unserem Leben „meint“ immer etwas. Insofern gibt es keine Sprache ohne irgendeine Bewusstseins-Dimension.

- Was meine ich nun mit dem Bewusstsein des „Unsagbaren“?

- Worum geht es hier? 
Edmund Husserl spricht in seiner „Phänomenologie“ davon „was sich (phänomenal) zeigt“ (Husserl 1985). Das ist der intentionale Gegenstand in Bewusstsein und auch in der Sprache.

Was ist aber nun dann die Situation, wenn wir das, was sich zeigt, auf keinen Begriff, keinen sprachlichen Ausdruck bringen können? Dann ist unser phänomenales Erleben und das, was wir darüber sagen können, ganz verschieden? Es gibt dann eine „Sprachkrise“, auch eine Art Bewusstseinskrise. Eine Störung der Kohärenz in unserem geistig-seelischen Erlebensfeld („dem Rahmen“), in unserem Dasein.

\section{Neurobiologie des Bewusstseins}

Im neurobiologischen Verständnis hat Bewusstsein immer mit Wahrnehmung zu tun.

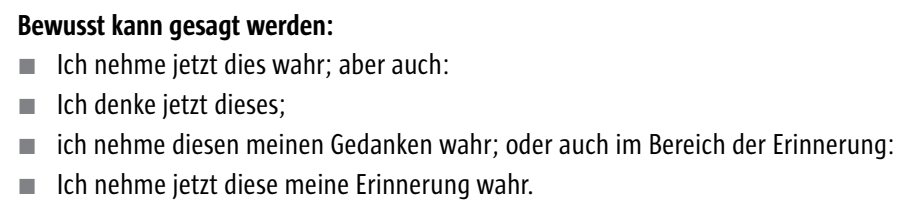

Die für mich überzeugendste Darstellung einer möglichen Neurobiologie des Bewusstseins stammt von dem bedeutenden Neuropsychologen Geoffrey Gray in seinem Buch „Consciousness“ (Gray 2004). Er geht davon aus, dass - neurobiologisch gesehen - Bewusstsein nur entsteht durch „Kontextualisierung und Integration“: Ich nehme jetzt dies wahr im Hinblick auf anderes. Kontext, Situation, Ereignis. Das stete Wahrnehmen des Gleichen ohne Variationen, ohne Veränderungen, wird, wie der britische Neurobiologe Donald Mc Kay gezeigt hat, sofort gelöscht. Es ist kein Ereignis mehr da, und so wird es auch aus dem Bewusstsein „weggeschoben“. Donald Mc Kay machte ein Experiment mit Botulinum-Toxin Injektionen in die Augenmuskeln. Wenn die Augenmuskeln gelähmt sind und der Kopf nicht bewegt wird, verschwindet der Seherfahrungsraum, verschwindet gewissermaßen „die Welt“ (zumindest die visuelle).

Geoffrey Gray ging nun von dem Phänomen der Angst aus. Er erforschte die Kontextualisierungsprozesse, die erforderlich sind, um Angst zu empfinden.

Der entscheidende Durchbruch, der Gray im Verständnis von Bewusstsein (in Angst) gelang, ist der Gedanke der „Fehlerkorrektur im letzten Moment“. Er schreibt dazu Folgendes:

"Consciousness - creeping up on the hard problem": "So what survival value can be found for consciousness, within the constraints outlined above? To answer this question I have proposed that consciousness acts as a late detector of error."

Mit anderen Worten: Es geht um das Phänomen der „Entscheidung“ (im Bewusstsein) im Sinne einer Neurobiologie der Freiheit. Dies im Hinblick auf die Philosophie der Freiheit und die nach Wolf Singer und Gerhard Roth angebliche Autonomie des Gehirns, welches - als verfügend über das Subjekt - soviel Furore gemacht hat (Allmacht des Gehirns).

Nach Gray geht es dabei um das Phänomen des sich Erinnerns: Der Hippocampus als ein Zuordnungssystem im Hinblick auf die Erinnerungsspeicher im Cortex wird hier funktionell aktiv (s. Abb. 4). Es wird neuropsychologisch die Frage bearbeitet: Wie 


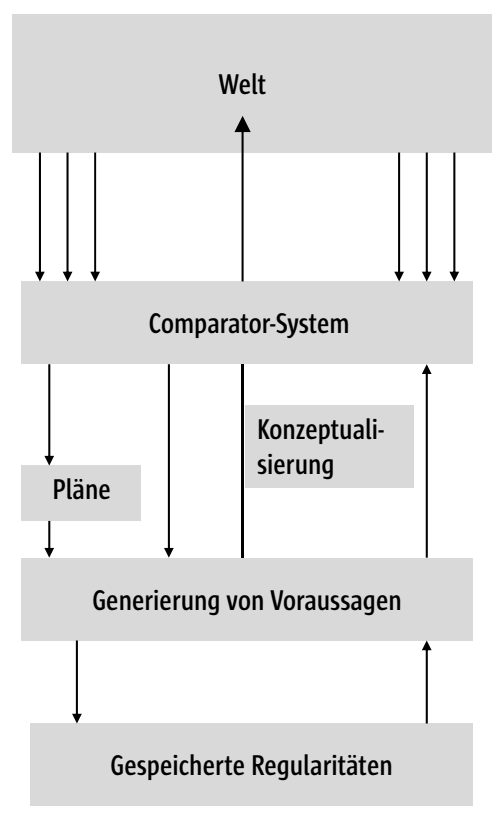

Abb. 4 Darstellung des „hippocampalen Comparator" Systems nach Gray und Rawlins, wobei jeweils Abgleiche zwischen Außendaten und gespeicherten Innendaten (stored regularities) durchgeführt werden

ist eine ähnliche Situation bisher ausgegangen? Das System hat dies (im Sinne von G. Roth und W. Singer) bereits „Vor-entschieden“. Aber der „bewusste Akt der Entscheidung“ hat noch einmal die Chance, einzugreifen und nach dem jetzt gerade vorliegenden Kontext, der „Situation“- der Gesamtheit der verfügbaren Daten - interaktiv nun noch einmal neu und endgültig zu entscheiden, was geschehen soll. Das ist nach Gray die Wurzel von bewusstem Bewusstsein: Aus einer gefährlichen Situation heraus wird korrigiert. Bei diesen Entscheidungen, quasi „aus dem letzten Moment heraus“, werden durchaus auch Impulse aus dem Unbewussten integriert, z.B. verschüttete Trauma-Erinnerungen, „subliminale Anteile“ des Erlebens in der Vergangenheit aber auch Clücksmomente und gutes Gelingen.

\section{Einheit und Multiplizität von Bewusstsein - das Ich und das Wir im Bewusstsein: das Ich als ein Wir (ein Paradox)}

Dass die Einheit von Bewusstsein keine ganz unproblematische und gewissermaßen frei Haus gelieferte Selbstverständlichkeit des sich selbst setzenden und sich selbst erfahrenden und reflektierenden Bewusstseins ist und sein kann: Diese Erkenntnis ist nicht so ganz neu und verdankt sich nicht der Moderne und der Postmoderne sondern bereits der Romantik, wo an allen kulturhistorischen Ecken und Enden und in 1.ooo-en von Variationen und Ausschmückungen von der Vielfältigkeit und Widersprüchlichkeit, der inneren Zerrissenheit und dem Doppelgängertum des Ich die Rede ist, sodass bereits in der Romantik - etwa von E.T.A. Hoffmann in den „Elixieren des Teufels“ (Hoffmann 1995) und von H.v. Hofmannsthal im „Andreas“-Roman (von 
Hofmannsthal 1982) - eher von dem Ich als einem Wir im Bewusstsein gesprochen werden kann als von gegebener Ich-Kohärenz als einer Einheit. Damit ergibt sich die in der Romantik, z.B. schließlich bei Dostojewskij im Doppelgänger-Roman (Dostojewskij 1990), auftauchende Frage:

In welcher Weise können wir unser Ich als ein Wir, quasi als eine Familie von „Bewussteins“ (im Plural), von Bewusstseinszuständen, erleben und vollziehen?

Hierbei steht der romantische Dichter und Philosoph - exemplarisch Hegel - vor der Aufgabe der Lösung des Paradoxons im Ich-Bewusstsein - der Philosoph Dieter Henrich sagt: „im Selbstverhältnis“ des Menschen (Henrich 1982) - das sich folgendermaßen formulieren lässt:

Das Ich-Bewusstsein, als Selbst-Bewusstsein, wird stets in der Paradoxie erlebt, dass einerseits das Selbst - als ein Ich - nur gedacht werden kann, radikal, als Einheit, da sonst Moralität nicht möglich ist.

Niemand ist verpflichtet, ein Versprechen, das er als ein anderer gegeben hat, zu halten, wenn es keine Kontinuität und Einheit der Person gibt - dass aber andererseits wir Menschen im Selbstumgang uns selbst nur verstehen und uns annehmen können unter der Bedingung des Ich als ein Wir, d.h. unter der Bedingung, dass wir uns selbst als eine Familie von Bewusstseins auffassen. Dies deshalb, weil wir sonst mit unseren verschiedenen Selbst-Identifikationen und Rollen-Identitäten nicht zurechtkommen, diese nicht beschreiben können, uns zu ihnen nicht in ein adäquates Verhältnis setzen können.

Von dieser Warte aus, unter dem Eindruck der Wucht dieser Paradoxie, erscheint Selbst-Kohärenz, Einheit von Bewusstsein, nicht als etwas quasi frei Haus geliefertes genuin Gegebenes, sondern als ein schwer zu erringendes kostbares Gut, zu dem Menschen durch viele Identitäts- und Reifungskrisen hindurch sich vorarbeiten können und vielleicht müssen; und die Forderung von Friedrich Nietzsche: „Werde der, der du bist“ hat dann eben gerade den Sinn, zu dieser Art der Selbstdurchdringung der Person aufzufordern, die zu so etwas wie Selbstkohärenz im Subjekt führt.

Wie aber sieht diese Selbstfindung aus im Hinblick auf Trauma und Krise?

Bevor ich mich dieser Frage weiter nähere, möchte ich die Bedeutung der hier erarbeiteten Tatsache beleuchten, dass im Selbstbewusstsein des Menschen eine fundamentale Paradoxie stets vorhanden ist; dies anhand der Frage:

Wie gehen wir Menschen normalerweise mit Paradoxien - mit unauflösbaren Widersprüchen um?

In der Physik, im Welle-Teilchen-Dualismus, müssen wir Elektronenwolken von Atomen und Molekülen zugleich als lokale Korpuskeln und als verteilte Wellen denken, ein Widerspruch, den wir dadurch auflösen, dass wir sagen, dass hier die Anschauung versagt. In der Frage des Selbstverhältnisses aber ist es nun so, dass Menschen auf diese Weise fundamental nicht beruhigt werden können; sie insistieren auf der Unvereinbarkeit der Doppelperspektiven, sie verfallen in tiefe Angst und existentielle Sorge. Der Philosoph Odo Marquardt sprach einmal davon, dass Philosophie mit einer besonderen Form von Paradoxie-Toleranz zu tun habe: glückliche Philosophen! 


\section{Der „Weltinnenraum“ als beseelter Raum: das Unbewusste „weiß“ („unbewusstes Bewusstsein“)}

Durch alle Wesen reicht der eine Raum: Weltinnenraum.

Dieses Zitat verdanke ich einer Anregung von Herrn Dr. Galuska: „Weltinnenraum“. Diesen Begriff hat der große philosophische Dichter Rainer Maria Rilke im Jahre 1914 geprägt (Rilke 1914). Sein Gedicht „Es winkt zur Fühlung fast aus allen Dingen“ erschließt uns fundamental die Bewusstseinsperspektive von allem „was ist“ und zeigt uns, dass ein radikaler Materialismus kein hinreichendes Weltbild ermöglicht, um mit der Existenz zurechtzukommen. Das Gedicht beginnt mit dem eigentümlichen Phänomen der Anmutung, einem Ruf, einem „Winken“, wo gesagt wird:

Es winkt zur Fühlung fast aus allen Dingen,
aus jeder Wendung weht es her: Gedenk!
Ein Tag, an dem wir fremd vorübergingen,
entschließt im künftigen sich zum Geschenk.
O Haus, o Wiesenhang, o Abendlicht,
auf einmal bringst du's beinah zum Gesicht
und stehst an uns, umarmend und umarmt.
Durch alle Wesen reicht der eine Raum:
Weltinnenraum, Die Vögel fliegen still
durch uns hindurch. O, der ich wachsen will,
ich seh hinaus, und in mir wächst der Baum.
Ich sorge mich, und in mir steht das Haus.
Ich hüte mich, und in mir ist die Hut.
Geliebter, der ich wurde: an mir ruht
der schönen Schöpfung Bild und weint sich aus.

Für Rilke ist gewissermaßen alles was ist mit so etwas wie einem „Weltinnenraum“ in Verbindung, es ist eine Innerlichkeitsdimension, die beinhaltet, dass, wie ich im meinem Buch „Texte zu Rilke“ sage, dass

Menschen, die an diesen Weltinnenraum nicht nur Anschluss haben (das haben wir nach Rilke alle), sondern über diese ihre eigene Verfasstheit auch Kenntnis haben, sich mit ihrem Anteil von Weltinnenraum auch in ein adäquates Verhältnis setzen, ihn sich also bewusst machen, aufhören ,einsam'zu sein. Sie sind eingebettet in einen wenn man so will, Innerlichkeitskosmos', der alles was ,ist' mit allem anderen verbindet, alle Momente des Daseins gewissermaßen im Hegelschen Sinne miteinander vermittelt (,aufhebt') und damit eine ,tröstende' Funktion hat (Emrich 2008).

Denn, wie Rilke in dem Gedicht sagt, wird hierdurch sogar ein Tag, an dem wir fremd vorübergingen, künftigem zum Geschenk.

Für uns Psychiater und Psychotherapeuten ist der wenn man so will „Innerlichkeits“Kosmos des „Weltinnenraums“ eine philosophisch-dichterische Beschreibung dessen, was wir „Psyche“ nennen; und es stellen sich die Fragen:

- Wie verhalten sich Psyche und Bewusstsein/Bewusstheit zueinander?

- Ist die Psyche uns (immer) oder überhaupt je „bewusst“?

- Ist Psyche nicht quasi universell und steckt in uns, in unserer Totalität?

- In Haut und Knochen, in Muskeln und Gelenken, im Herzen und im Gehirn, im Geist und dem inneren „Unentdeckten“, im Unbewussten? 
Um diese Fragen zu klären, bedarf es eines Verstehens, was wir mit Bewusstsein meinen und wieso es etwas gibt, dass wir das „unbewusste Bewusstsein“ nennen könnten. Das unbewusste Bewusstsein kann man auch als ein latentes Bewusstes auffassen, als etwas, was unerschlossen in uns wirkt und in einem „dynamischen Prozess" auf uns zukommen mag, sich uns erschließen mag oder auch im Verborgenen, Verdrängten, Abgespalteten zurückbleibt. Dabei kann die nur latent bewusste Psyche „mächtig“ werden; ich spreche in einem kürzlich im Jung-Journal veröffentlichten Text von der „Allmacht“ der Psyche (Emrich 2012) und belege dies anhand des Traums in Heinrich von Kleist's „Käthchen von Heilbronn“ anhand eines Traum-Bewusstseins, wo das „Käthchen“ Folgendes sagt:

Als ich zu Bett' ging, da das Blei gegossen,

In der Sylvesternacht, bat ich zu Cott,

Wenn's wahr wär', was mir die Mariane sagte,

Möcht' er den Ritter mir im Traume zeigen.

Und da erschienst du ja, um Mitternacht,

Leibhaftig, wie ich jetzt dich vor mir sehe,

Als deine Braut mich liebend zu begrüßen.

Das Käthchen wird durch die Macht des (mit dem Grafen von Strahl gemeinsamen) Traumes zur legitimierten Kaisertochter und Ehefrau des Grafen hin entwickelt.

Das Unbewusste bringt gewissermaßen sein „Votum“ ein; will etwas erreichen. Das Unbewusste kann sogar gegen das Wachbewusstsein ankämpfen, regelrecht „putschen“, gegen dieses „anrennen“. Es kann den Träger des Wachbewusstseins, das „Selbst“, zu „wecken“ versuchen: durch eine Art Anrufung, einen „Ruf“. In Kafkas Roman „Der Prozess“ ist es der Gefängnisgeistliche, der Josef K. anruft: „Josef K.“ (Kafka 1983). Das Ziel des Anrufes ist, wie ich in meiner Einleitung zu Wiebrecht Ries' Kafka-Buch sage (Ries 2011), zu erreichen, dass Josef K. sein Bewusstsein erweitert und eine „Intuition“ für sich selbst und für andere entwickelt, d.h. dass er vor seinem ,inneren Gericht“ bestehen kann und nicht verurteilt wird. Aber es steht, wie es in dem Roman heißt, „schlecht“ um seinen (inneren) Prozess. Er leugnet hartnäckig die Notwendigkeit seiner inneren Wandlung, seiner inneren „Öffnung“, im Sinne eines Wissens über die Fakten des Lebens hinaus.

Im Berufsleben sagt Josef $\mathrm{K}$. hätte mir ein solcher (innerer) Überfall, wie ihm dieser an seinem 30. Geburtstag passiert ist, nie geschehen können:

In der Bank zum Beispiel bin ich vorbereitet, dort könnte miretwas Derartiges unmöglich geschehen, ich habe dort einen eigenen Diener, das allgemeine Telefon und das Bürotelefon stehen vor mir aufdem Tisch ... vor allem bin ich dort immerfort im Zusammenhang der Arbeit, daher geistesgegenwärtig.

Das unbewusste Wissen ist mächtig und gerät in „Resonanz“ mit Erlebnissen im Laufe des Lebens-Schicksals, z.B. in Heinrich von Kleists Novelle „Die Heilige Cäcilie oder die Gewalt der Musik“.

Demnach kam es wie ein wunderbarerhimmlischer Trost in die Herzen der frommen Frauen; siestellten sich augenblicklich mit ihren Instrumenten an die Pulte; die Beklemmung selbst, in der sie sich befanden, kam hinzu, um ihre Seelen wie aufSchwingen durch alle Himmel des Wohlklangs zu führen: das Oratorium ward mit der höchsten und herrlichsten musikalischen Pracht ausgeführt. 
... es regte sich während der ganzen Darstellung kein Odem in den Hallen und Bänken; besonders bei dem salve regina und noch mehrbei dem gloria in excelsis war es, als ob die ganze Bevölkerung der Kirche tot sei: dergestalt, daß, den vier gottverdammten Brüdern und ihrem Anhang zum Trotz, auch der Staub auf dem Estrich nicht verweht ward ...

Es kommt hier zu Resonanzphänomenen und diese sind auch Gegenstand von Psychotherapien. Derartige Resonanzphänomene sind allfällig, ständig, im Leben vorhanden und prägen uns (mimetisch); sie können uns voranbringen, Reifungskrisen hervorrufen oder aber auch uns traumatisieren, herausfordern oder auch schwer zurückwerfen (wie z.B. in dem zweiten Teil der Kleist Novelle zur Gewalt der Musik, in der die verhinderten Bilderstürmer eine Art dämonisches Besessenheitsritual in der psychiatrischen Klinik, in die sie eingewiesen worden waren, austoben müssen), das ich als eine quasi „erfundene Zwangskrankheit“ folgendermaßen beschrieben habe:

So fangen sie mit einer entsetzlichen und grässlichen Stimme das gloria in excelsis zu intonieren an. So mögen sich Leoparden und Wölfe anhören lassen, wenn sie zur eisigen Winterzeit das Firmament anbrüllen: die Pfeiler des Hauses, versichere ich Euch, erschütterten, und die Fenster, von ihrer Lungen sichtbarem Atem getroffen, drohten klirrend, als ob man Hände voll schweren Sandes gegen ihre Flächen würfe, zusammenzubrechen. Bei diesem grausenhaften Auftritt stürzen wir besinnungslos mit sträubenden Haaren auseinander: wir zerstreuen uns, Mäntel und Hüte zurücklassend ...

\section{Transzendierendes Bewusstsein}

Transzendierendes Bewusstsein ist eine Form des „Selbstüberstieges“; die Philosophen nennen dies die „Selbsttranszendenz“ oder auch - als Vollzug - „Selbsttranszendierung“. Das Bewusstsein holt sich in seiner Selbstreflexivität gewissermaßen selbst ein und erreicht eine höhere Form von Bewusstseinsfeld, das für die Therapie von entscheidender Bedeutung ist.

Psychotherapien versuchen nun, die Kohärenz zwischen den Impulsen, die vom Wissen (und Wollen) des Unbewussten ausgehen und dem bewussten Wissen zu erhöhen und damit einen besseren Kompromiss zu erreichen, d.h. einen Ausgleich zwischen den im Traumbewusstsein aufscheinenden archetypischen inneren Wesenheiten und Gestalten und dem Bewusstsein der Wachheit. Man beschreibt zu Recht Neurosen als „suboptimale Kompromissbildungen“ zwischen Lebensanforderungen und inneren unlösbaren Konflikten. In meinem Text zur Heiligen Cäcilie beschreibe ich diese Option, den Kompromiss zu verbessern und die Kohärenz zu erhöhen, in folgender Weise:

Nach der Konzeption, dass Zwangsrituale mit der Frage der Psyche zu tun haben: „Warum tue ich eigentlich nicht, was ich will?“ (Emrich 2002) wird die (scheinbar entlastende) Antwort der Psyche gegeben: Es ist der Zwang, der mich an meiner eigenen (einsichtsvollen) Spontaneität des Willens und dessen Ausführung hindert. Insofern hat das Zwangsritual etwas Entlastendes. So auch hier bei Heinrich von Kleist: das Zwangsritual, als Gruppe jeden Tag um eine bestimmte Uhrzeit in einer grausigen unmusikalisch-heulenden Form die beschriebene Antimusik zur heiligen Cäcilie herauszuschreien, hat eine (in der Sackgasse der Unvernunft notwendige) haltgebende, entlastende Funktion. Eine (therapeutische) Chance wäre die Einsicht: Was wir damals wollten, war falsch. Die „Gewalt“ dieser Antimusik, die zugleich Traumabe- 
wältigung im Sinne der „Wieder-holung“ (Kierkegaard) und haltgebende Funktion im Ritual der Zwangserkrankung darstellt, hat den Sinn, letztlich mit dem Identitätsverlust des Scheiterns als Bilderstürmer fertig zu werden.

Fragen nach dem Ursprung von Gewalt sind in bestimmter Hinsicht immer auch Identitätsfragen. Deshalb kann man auch von einer Dynamik, einer Energie, der „Identitäts-Bildungsenergie“ sprechen (Emrich, 2007).

„Für Freud ist also diese Tendenz zur Einheitlichkeit des Bewusstseinseine wesentliche Quelle von energetischer Aufladung der Psyche. In selbstpsychologischer Nomenklatur bedeutet dies, dass die Entwicklung von, interner Kohärenz' nur durch Aufwendung erheblicher intrapsychischer Energien geleistet werden kann, sodass die Identitäts-Bildungsenergie auch als ,Kohärenzenergie‘ oder auch als, Ich-strukturelle Bindungsenergie‘ zu bezeichnen ist. Da diese Form von Energie letztlich darauf zurückgeht, dass widersprüchliche Identifikationen zu bewältigen sind, kann man sie auch als, mimetische Energie' in Anlehnung an Girard bezeichnen. Die Bildung von Identität geht immer mit schmerzhaften Opferungen von bisher positiv besetzten Selbstanteilen einher, und insofern ist ,Opfer' eine rituelle Repräsentation von Identitätsbildungsprozessen, die sich im kommunikativen Raum des ,Zwischen', d.h. einer Sphäre abspielen, die bereits vorgängigZwang zum ,Kompromiss', Zwang zur Selbstrelativierung und damit ,Selbstentäußerung an das Gemeinsame bedeutet."

Eine besondere Möglichkeit, hier durch intensive Lebensereignisse und Rituale, eingreifende Therapiemethoden und quasi „kathartische Therapien“ voranzukommen, ist der therapeutische Umgang mit dem „ekstatischen“ Bewusstsein bzw. durch die Anwendung des „holotropen Bewusstseins“, das durch eine besondere Atemtechnik entstehen kann, wie es von Stanislaw Grof beschrieben wurde. Grof hat vor zwei Wochen in Wien einen interessanten Vortrag gehalten, in dem er von einer „Technologie des Heiligen“ und einem Bewusstsein der „holotropen Zustände“ gesprochen hat, einem mentalen Bewusstseinsgeschehen, das, wie er sagt „auf das Ganze hin orientiert“ ist. Er beschreibt seine erste LSD-Erfahrung, die er als Ausbildungsmittel für Psychiater und Psychotherapeuten in Anwendung brachte, als „gigantisches Licht“ und als eine paradoxe Erfahrung, ein Alles und Nichts zugleich, eine Anwesenheit des Kosmos, wobei er darauf hinweist, dass holotrope Zustände auch spontan als Episoden des Bewusstseinsstroms auftreten können.

Die Wurzel derartiger Erfahrungs- und Erlebnis- und Gedankenwelten liegt im Bereich der sog. „abnormen Bewusstseinszustände“ (altered states of consciousness), wie sie unter experimentellen Drogen wie LSD und Meskalin erforscht wurden, die aber ein Wissens-Kulturgut seit der Entstehung der Menschheitsgeschichte und Menschheitskultur darstellen; dies in Religionen, Ritualen, Festen und Therapien durch Priester und Schamanen. Es geht um Bewusstseinserweiterung und Bewusstseinsrefokussierung, wie von Stanislaw Grof eindrucksvoll beschrieben.

Eine weitere Methode, mit Hilfe derer auch neurophysiologisch/neuropsychologisch plausibel gemacht werden kann, was sich da eigentlich ereignet, ist das Atmen mit Hyperventilation, das holotrope Atmen, wobei ein Bewusstseinszustand hervorgerufen wird, der wie gesagt auf „das Ganze gerichtet“ ist. Prof. Passie beschreibt dies in seinem exzellenten Buch „Ekstase“ in folgender Weise:

Bei der Hyperventilation nimmt die Hirndurchblutung aufgrund einer starken Verminderung des Kohlendioxidgehaltes ... stark ab. ... Dies impliziert, dass die darunter liegenden Hirnschichten, die sog. subcortikalen Bereiche, relativ stärker durchblutet werden und somit an der Ausgestaltung des Erlebens stärker beteiligt sein dürften. 
Einen Beitrag hierzu liefert auch die „sanfte Katharsis“: Die griechische Tragödie als bewusstseinsverändernde therapeutische Einrichtung wurde von Aristoteles beschrieben als eine Form der „Reinigung des Cemütes“, der Psyche, der Seele. In diesem Sinne hat das Drama eine kathartische bewusstseinserweiternde und bewusstseinsverändernde Dimension. Im Sinne der Logotherapie von Viktor Frankl kann man dabei auch davon sprechen, dass anhand der archetypischen Schicksals-Darstellungen in der Tragödie die eigene Schicksalhaftigkeit des Lebens erspürt werden kann und damit eine bessere Kompromissbildung zwischen unbewussten Konflikten und dem realen Erleben möglich wird.

\section{Auf dem Wege zum transzendierenden Verzicht auf das „Meinen von etwas“; dies im Sinne eines „spirituellen Bewusstseins“}

Im „absoluten Bewusstsein“, dem, was wir in psychedelischen Bewusstseinszuständen, holotropen, ekstatischen, spirituellen Verfasstheiten erleben, gibt es kein konkret fassbares Bewusstseinssubjekt mehr, von dem gesagt werden könnte, dass das „Haben von Bewusstsein“ ihm zugeschrieben werden kann, ihm inhärent sei. Vielmehr ist es eine höherstufige Bewusstseinsform, die sich quasi der Totalität des Daseins verdankt, eine Art von „Weltgeist“, von „absolutem Geist“. Hier geht es um Bewusstseinszustände, die keine konkreten Bewusstseinsinhalte mehr zum Gegenstand haben und sich auf „das Ganze“ bzw. auch das „Nichts“ beziehen. In diesem Denken, das man auch das Zarathustra-Denken Friedrich Nietzsches nennen kann, ist das Resultat der Gesamtheit aller Vorgänge die „ewige Wiederkehr des Gleichen“. Dies als eine irreversible, zeit-unabhängige Totalität als Untergrund (Hypokeimenon) des Daseins. Um das Verständnis dieser radikalen Konzeption Nietzsches, die er als den radikalen Durchbruch seines Geistes erlebt hat, begreiflich zu machen, scheint es wichtig, sich zu verdeutlich, dass dieser neuartige Gedanke nicht beinhaltet: Die ewige Wiederkehr von „genau dem Gleichen“ im materialistisch physikalischen Sinne, sondern in dem Sinne: „Im Grunde passiert immer wieder dasselbe“. Dies als ein durchaus tröstliches und dem „Weltwissen“ nahekommendes Bewusstseinsphänomen. Das Erreichen eines solchen Bewusstseinszustandes höchster Transzendierung wird quasi „zwischen den Zeilen“ auch in Sigmund Freuds Gedanken eines „Nirvana“-Bewusstseins konzeptualisiert, das Freud auch versuchsweise mit seinem Konzept des „Todestriebes" in Verbindung gebracht hat. Dieser etwas heiklen Konstruktion habe ich vor einigen Jahre eine neue Deutung gegeben, indem ich von Henri Bergsons zeitphilosophischer Konzeption ausging, in der das Vergangene unwiderruflich in die Gegenwart hereinragt im Sinne seines Satzes: „Die Vergangenheit ist nicht vergangen“; mit anderen Worten: Es gibt eine Art Ewigkeitscharakter des Vergangenen im Hinblick auf die Gegenwart. Dies kann man zusammen mit Marcel Prousts Erinnerungs- und Vergangenheitskonzept in folgender Weise auf den Todestrieb-Gedanken anwenden: „Nirvana-Bewusstsein“ ist als ein Bewusstsein der Auslöschung aller erinnerten Konkretheiten so zu sehen, dass der Gedanke: „Die Vergangenheit ist nicht vergangen“ durch einen Vorgang des „vergegenwärtigenden Vergessens“ Todestrieb-artig umgekehrt wird. Es kommt zu einer Art „Auslöschen“ der Wirkmacht der Vergangenen auf einer höheren Bewusstseinsstufe. Dadurch kommt es auch zu einer Versöhnung mit dem traumatisch Prägenden, das - wie der große Zeitphilosoph Michael Theunissen sagt - als die „Herrschaft der Zeit über die Gegenwart“ - das Leben beherrscht und durchherrscht hatte. Das Denken, Wollen, Leben und Fühlen enthält sich des „Meinens von etwas“ und verliert - im Sinne Husserls - seine „intentionalen Gegenstände“. 
Es kommt zu einer Annäherung an Hegels „absolutes Bewusstsein“, das quasi alles mit allem ,aufhebt“ und somit das Subjekt über die Immanenz des Seienden hinaushebt in einen Zustand von „Frieden“, Versöhnung, Transzendierung.

Der so gedeutete „Todestrieb“, der kein Trieb ist sondern das Resultat einer Weisheitserfahrung, die zu Erlösung und Erleuchtung führt, erlaubt - durch das Auslöschen des traumatisch-Immanenten der Vergangenheit - die Transzendierung hin zu einer höheren Bewusstseinsstufe von Heilung und Existenz.

\section{Literatur}

Dostojewski FM (1990) Der Doppelgänger. Piper Verlag München

Emrich HM (2008) Texte zu Rilke. Cuvillier Verlag Göttingen

Emrich HM (2012) Allmacht der Psyche. Jung Journal 15(28), 25-28

Fichte IG (1979) Grundlage der gesamten Wissenschaftslehre: als Handschrift für die Zuhörer (1794). Meiner Verlag Hamburg

Freud S (1914) Die Anfänge und Begründung der Psychoanalyse durch Freud: Die Lehre von der Verdrängung und vom Widerstand. URL:

http://www.textlog.de/freud-psychoanalyse-lehre-verdaengung-widerstand.html (abgerufen am 05.06.2013)

Goffman E (1977) Rahmenanalyse (Frame Analysis). Suhrkamp Verlag Frankfurt a.M.

Gray I (2004) Consciousness - Creeping up on the Hard Problem. Oxford University Press USA

Henrich D (1982) Selbstverhältnisse. Reclam Verlag Stuttgart

Hoffmann ETA (1995) Die Elixiere des Teufels. Goldmann Verlag München

Hofmannsthal Hv (1982) Andreas. In: M. Pape (Hrsg.) Sämtl. Werke, Band 30, Fischer Verlag Frankfurt a.M.

Husserl E (1985) Texte zur Phänomenologie des Inneren Zeitbewußtseins (1893-1917). Felix Meiner Verlag Hamburg

James W (1890) The priciples of psychology. Macmillan London

Kafka F (1983) Der Prozeß. Fischer TB Verlag Frankfurt

Kane S (2002) Sämtliche Stücke. Rowohlt Verlag Hamburg

Kant I (1974) Kritik der reinen Vernunft. Suhrkamp Verlag Frankfurt a.M.

Kleist Hv (1994) Die heilige Cäcilie oder die Gewalt der Musik. In: H. Sembdner (Hrsg.) Sämtliche Werke und Briefe. In zwei Bänden. Deutscher Taschenbuch Verlag München

Mackay DM (1965) A mind's eye view of the brain. In: Wiener N u. Schade JP (eds.). Progress in Brain Research, Vol. 17: Cybernetics of the Nervous System. 321-332. Elsevier Amsterdam

Passie T, Belschner W (Hrsg.) (2008) Ekstase: Phänomen - Erfahrung - Heilung. Internationalismus-Verlag Hannover

Proust M (1993) Auf der Suche nach der verlorenen Zeit. Suhrkamp Verlag Frankfurt a.M.

Ricoeur P (1991) Zeit und Erzählung. Bd. 3: Die erzählte Zeit. Fink Verlag München

Rilke (1998) Die Gedichte. Insel Verlag Frankfurt a.M.

Rilke RM (1997) Die Aufzeichnungen des Malte Laurids Brigge. DTV München

Schafer R (1982) Eine neue Sprache für die Psychoanalyse. Klett-Cotta Verlag Stuttgart

Theunissen, M (1992) Negative Theologie der Zeit. Suhrkamp Verlag Frankfurt a.M.

Whitehead AN (1979) Prozeß und Realität. Suhrkamp Verlag Frankfurt a.M.

Ries W (2011) Maskeraden des Auslands. Lektüren zu Franz Kafkas „Process“. Elfenbein Verlag Berlin

Urban K (1998) Vortrag auf der Tagung der Gesellschaft Deutscher Naturforscher und Ärzte Gene, Neurone, Qubits und $\mathrm{Co}$. Unsere Welten der Information, Berlin

Wittgenstein L (1984) Werkausgabe in 8 Bänden, Bd. 5: Das Blaue Buch. Suhrkamp Verlag Frankfurt a.M.

Woolfe, V (1994) Die Wellen. Fischer Verlag Frankfurt a.M. 


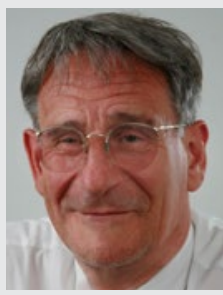

Prof. em. Dr. med. Dr. phil. Hinderk M. Emrich

Arzt und Professor für Neurologie und Psychiatrie/Klinische Pharmakologie, Psychotherapeut, Psychoanalytiker; von 1992 bis 2008 Leiter der Klinik für Psychiatrie, Sozialpsychiatrie und Psychotherapie der Medizinischen Hochschule Hannover.

Lehrauftrag an der Deutschen Akademie für Film und Fernsehen; Promotion in Philosophie 1999; Venia legendi für Philosophie an der Universität Hannover seit 2002.

Wesentliche Forschungsgebiete: Psychopharmakologie, Wahrnehmungspsychologie und Systemtheorie von Psychosen, Synästhesie.

Zusätzliche wissenschaftliche Interessen: analytische Philosophie des Geistes, Psychoanalyse nach C.G. Jung, Medientheorie, Tiefenpsychologie des Films. 